\title{
Comparative Efficacy of Some Bio-Rational and Chemical Insecticides against Whitefly Bemisia tabaci (Gennadius) on Okra Abelmoschus esculentus (L.) Moench
}

\author{
Sumit Kumar Singh* and Sasya Thakur
}

Department of Entomology, Sam Higginbottom University of Agriculture, Technology and Sciences, Allahabad - 211 007, India

*Corresponding author

\begin{tabular}{|c|c|}
\hline & B \\
\hline & \multirow{6}{*}{ 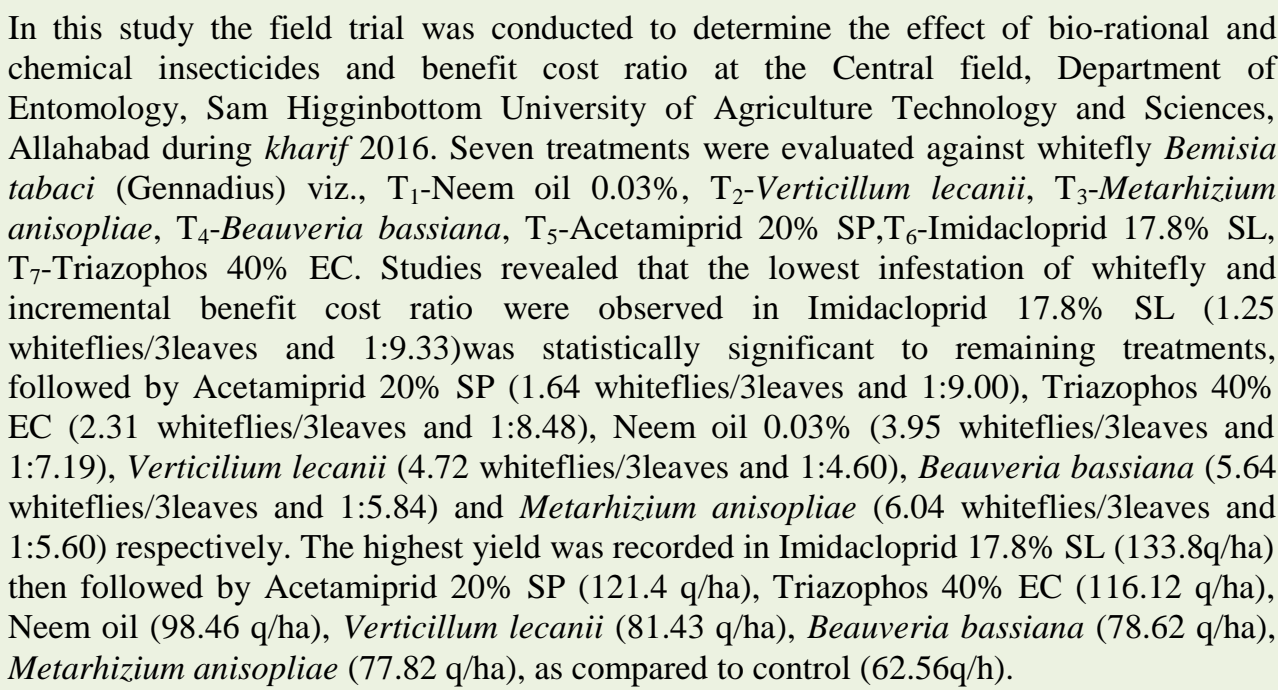 } \\
\hline Keywor & \\
\hline & \\
\hline Artic & \\
\hline & \\
\hline & \\
\hline
\end{tabular}

\section{Introduction}

Among the vegetable crops grown in India, Okra (Abelmoschus esculentus L. Moench), also known as lady's finger or bhendi, belongs to family Malvaceae and is an important crop grown throughout the year. Besides India, it is grown in many tropical and subtropical parts of the world. Tender fruits are used as vegetables or in culinary preparations as sliced and dried pieces. It is also used for thickening gravies and soups, because of its high mucilage content. The roots and stems of okra are used for cleaning cane juice Chauhan (1972). Matured fruits and stems containing crude fibre are used in paper industry. It has good nutritional value, particularly the high content of Vitamin C (30 mg/100 g), Calcium $(90 \mathrm{mg} / 100 \mathrm{~g})$, Iron $(1.5 \mathrm{mg} / 100 \mathrm{~g})$ and other minerals like magnesium and potassium, Vitamin $\mathrm{A}$ and $\mathrm{B}$, fats and carbohydrates 
Aykroud (1963). Among 72 species of insects infesting the crop, the sucking pest viz., Aphids (Aphis gossypii Glover), leafhopper (Amrasca biguttula biguttula Ishida) and whitefly (Bemisia tabaci Gennadius) are the most important which cause significant yield reduction. Aphids and leafhopper are important pests in the early stage of the crop which desap the plants, making them weak which results in reduced flowering and poor fruit set ultimately reduction in yield. The cultivation of okra in India received a setback due to yellow vein mosaic virus (YVMV) and enation leaf curl virus (ELCV), spread by the vector whitefly. The loss in marketable yield has been estimated at 50-94\%, depending up on the stage of crop growth at which the infection occurs. Failure to control these pests in the initial stage causes a yield loss upto 54.04 per cent Chaudhary and Dadeech (1989). Thus in the present investigation an attempt has been made to evaluate the efficacy of some bio-rational and chemical insecticides against whitefly infesting okra along with benefit cost ratio.

\section{Materials and Methods}

The experiment was conducted during kharif season 2016 at the Central field of "Sam Higginbottom University of Agriculture, Technology and Sciences" Allahabad, Uttar Pradesh, India, laid in a Randomized Block Design (RBD) with three replications and eight treatments, using variety VNR-22 (Komal) in a plot size of $(2 \mathrm{~m} \times 1 \mathrm{~m})$ at a spacing of $(45 \times 30 \mathrm{~cm})$ with recommended package of practices. The insecticidal treatments include viz., $\mathrm{T}_{1}$-Neem oil $0.03 \%$, $\mathrm{T}_{2}$-Verticillum lecanii, $\quad \mathrm{T}_{3}$-Metarhizium anisopliae, $\mathrm{T}_{4}$-Beauveria bassiana, $\mathrm{T}_{5^{-}}$ Acetamiprid 20\% SP, $\mathrm{T}_{6}$-Imidacloprid $17.8 \%$ SL, $\mathrm{T}_{7}$-Triazophos 40\% EC along with untreated control. The spraying was done after the population reaching its ETL. Two insecticidal sprays were administrated at $38^{\text {th }}$
(SMW) and $41^{\text {st }}$ (SMW) starting from 42 days after sowing. The observations on the number of whitefly were made on the five randomly selected and tagged plants from each plot. The number of pest per leaf was calculated based on the number of insects on three leaves, each taken from top, middle and bottom of each plant. The observations are made 1 day before spray (DBS) followed by $3^{\text {rd }}, 7^{\text {th }}, 14^{\text {th }}$ days after spray (DAS). Observations were recorded without disturbing the plants to minimize the observational errors. Population of whitefly was recorded from each net plot and the population was worked out per leaf. Fruit yield was also recorded. The total yield of the marketable fruits obtained from different treatments was calculated and converted by considering the additional cost (cost of insecticides and operational charges) and benefit (compared to untreated control) in the respective treatments.

\section{Results and Discussion}

The infestation of whitefly revealed that the lowest infestation of whitefly was recorded in $\mathrm{T}_{6}$-Imidacloprid (1.25 whiteflies/3leaves) recorded lowest population of whitefly and was statistically significant to remaining treatments, followed by $\mathrm{T}_{5}$-Acetamiprid (1.64 whiteflies/3leaves), $\quad \mathrm{T}_{7}$-Trizophos $\quad$ (2.31 whiteflies/3leaves), $\quad \mathrm{T}_{1}$-Neem oil (3.95 whiteflies/3leaves), $\mathrm{T}_{2}$-Verticilium lecanii (4.72 whiteflies/3leaves), $\quad \mathrm{T}_{4}$-Beauveria bassiana (5.64 whiteflies/3leaves) and $\mathrm{T}_{3^{-}}$ Metarhizium anisopliae (6.04 whiteflies/ 3leaves) showed least effect among all other treatments (Table.3).

Similar readings were found with Preetha $e t$ al., (2009) showed among the newer insecticide molecules evaluated, efficacy of neonicotinoid, imidacloprid as foliar application against whitefly Bemesia tabaci. Imidacloprid 17.8 SL was found effective against whiteflies. The other neonicotinoid 
also provide similar level of protection as that of imidacloprid. These findings can be supported with the study of Begum et al., (2016) who reported that imidacloprid 17.8 SL was the most effective treatment indicating reduction in population of whiteflies. Over all best performance of insecticides against whitefly was recorded in imidacloprid treated plots with lowest population of whitefly (3.91 whitefly/15 leaves) by Sarkar et al., (2016). Ghosh et al., (2013) recorded satisfactory control $>60 \%$ population suppression with imidacloprid and neem oil.

Table.1 Effect of some bio-rational and chemical insecticides against whitefly, Bemisia tabaci (Gennadius) on okra Abelmoschus esculentus (L.) Moench (First spray)

\begin{tabular}{|c|c|c|c|c|c|c|}
\hline \multirow[t]{2}{*}{ Tr. No. } & \multirow[t]{2}{*}{ Treatments } & \multicolumn{5}{|c|}{ Number of Whiteflies / 3 leaves } \\
\hline & & $1 \mathrm{DBS}$ & 3 DAS & 7 DAS & 14 DAS & Mean \\
\hline $\mathbf{T}_{0}$ & Untreated (Control) & $\begin{array}{c}7.40 \\
(\mathbf{2 . 7 2})\end{array}$ & $\begin{array}{c}8.60 \\
(\mathbf{2 . 9 3})\end{array}$ & $\begin{array}{c}9.46 \\
(\mathbf{3 . 0 7})\end{array}$ & $\begin{array}{l}10.80 \\
\mathbf{( 3 . 2 8 )}\end{array}$ & $\begin{array}{c}9.62 \\
(\mathbf{3 . 1 0})\end{array}$ \\
\hline $\mathbf{T}_{1}$ & Neem oil $(0.03 \%)$ & $\begin{array}{c}7.53 \\
(\mathbf{2 . 7 4})\end{array}$ & $\begin{array}{c}4.46 \\
(\mathbf{2 . 1 1})\end{array}$ & $\begin{array}{c}4.13 \\
(\mathbf{2 . 0 3})\end{array}$ & $\begin{array}{c}5.60 \\
(2.36)\end{array}$ & $\begin{array}{c}4.73 \\
(\mathbf{2 . 1 7})\end{array}$ \\
\hline $\mathbf{T}_{2}$ & Verticillium lecanii & $\begin{array}{c}7.46 \\
(\mathbf{2 . 7 3})\end{array}$ & $\begin{array}{c}5.40 \\
(\mathbf{2 . 3 2})\end{array}$ & $\begin{array}{c}4.86 \\
\mathbf{( 2 . 2 0 )}\end{array}$ & $\begin{array}{c}5.93 \\
(\mathbf{2 . 4 3})\end{array}$ & $\begin{array}{c}5.40 \\
(\mathbf{2 . 3 2})\end{array}$ \\
\hline $\mathbf{T}_{3}$ & Metarhizium anisopliae & $\begin{array}{c}7.20 \\
(\mathbf{2 . 6 8 )}\end{array}$ & $\begin{array}{c}6.06 \\
(\mathbf{2 . 4 6})\end{array}$ & $\begin{array}{c}5.73 \\
(2.39)\end{array}$ & $\begin{array}{c}6.80 \\
\mathbf{( 2 . 6 0 )}\end{array}$ & $\begin{array}{c}6.20 \\
(\mathbf{2 . 4 9})\end{array}$ \\
\hline $\mathbf{T}_{4}$ & Beauveria bassiana & $\begin{array}{c}7.26 \\
(\mathbf{2 . 6 9})\end{array}$ & $\begin{array}{c}5.73 \\
(\mathbf{2 . 3 9})\end{array}$ & $\begin{array}{c}5.46 \\
(\mathbf{2 . 3 3})\end{array}$ & $\begin{array}{c}6.66 \\
(\mathbf{2 . 5 8})\end{array}$ & $\begin{array}{c}5.95 \\
(\mathbf{2 . 4 4})\end{array}$ \\
\hline $\mathbf{T}_{5}$ & Acetamiprid 20\% SP & $\begin{array}{c}7.40 \\
(\mathbf{2 . 7 2})\end{array}$ & $\begin{array}{c}1.80 \\
(\mathbf{1 . 3 4})\end{array}$ & $\begin{array}{c}1.66 \\
(\mathbf{1 . 2 9})\end{array}$ & $\begin{array}{c}3.06 \\
(\mathbf{1 . 7 5})\end{array}$ & $\begin{array}{c}2.17 \\
(\mathbf{1 . 4 7})\end{array}$ \\
\hline$T_{6}$ & Imidacloprid $17.8 \% \mathrm{SL}$ & $\begin{array}{c}7.46 \\
\mathbf{( 2 . 7 3 )}\end{array}$ & $\begin{array}{c}1.40 \\
\mathbf{( 1 . 1 8 )}\end{array}$ & $\begin{array}{c}1.13 \\
\mathbf{( 1 . 0 6 )}\end{array}$ & $\begin{array}{c}2.26 \\
\mathbf{( 1 . 5 0 )}\end{array}$ & $\begin{array}{c}1.60 \\
\mathbf{( 1 . 2 6 )}\end{array}$ \\
\hline $\mathbf{T}_{7}$ & Trizophos $40 \%$ EC & $\begin{array}{c}7.33 \\
(\mathbf{2 . 7 0})\end{array}$ & $\begin{array}{r}2.53 \\
(\mathbf{1 . 5 9 )}\end{array}$ & $\begin{array}{c}2.46 \\
(\mathbf{1 . 5 7})\end{array}$ & $\begin{array}{c}3.40 \\
\mathbf{( 1 . 8 4 )}\end{array}$ & $\begin{array}{c}2.80 \\
(\mathbf{1 . 6 7})\end{array}$ \\
\hline & F- test & NS & S & S & S & S \\
\hline & S. Ed. $( \pm)$ & 0.020 & 0.038 & 0.032 & 0.035 & 0.017 \\
\hline & C. D. $(P=0.05)$ & 0.060 & 0.091 & 0.084 & 0.088 & 0.060 \\
\hline
\end{tabular}

*Figures in parenthesis are square root transformed values. DBS-Days before spray, DAS-Days after spray. 
Table.2 Effect of some bio-rational and chemical insecticides against whitefly, Bemisiata baci (Gennadius) on okra Abelmoschus esculentus (L.) Moench (Second spray)

\begin{tabular}{|c|c|c|c|c|c|c|}
\hline \multirow[t]{2}{*}{ Tr. No. } & \multirow[t]{2}{*}{ Treatments } & \multicolumn{5}{|c|}{ Number of Whiteflies / 3 leaves } \\
\hline & & $1 \mathrm{DBS}$ & 3 DAS & 7 DAS & 14 DAS & Mean \\
\hline $\mathbf{T}_{0}$ & Untreated (Control) & $\begin{array}{c}11.73 \\
\mathbf{( 3 . 4 2 )}\end{array}$ & $\begin{array}{r}12.40 \\
\mathbf{( 3 . 5 2 )}\end{array}$ & $\begin{array}{c}11.73 \\
\mathbf{( 3 . 4 2 )}\end{array}$ & $\begin{array}{l}10.40 \\
\mathbf{( 3 . 2 2 )}\end{array}$ & $\begin{array}{l}11.51 \\
(3.39)\end{array}$ \\
\hline $\mathbf{T}_{1}$ & Neem oil $(0.03 \%)$ & $\begin{array}{c}8.33 \\
(\mathbf{2 . 8 8})\end{array}$ & $\begin{array}{c}3.46 \\
(\mathbf{1 . 8 6})\end{array}$ & $\begin{array}{c}3.13 \\
(\mathbf{1 . 7 6})\end{array}$ & $\begin{array}{c}2.93 \\
(\mathbf{1 . 7 1})\end{array}$ & $\begin{array}{c}3.17 \\
(\mathbf{1 . 7 8})\end{array}$ \\
\hline $\mathbf{T}_{2}$ & Verticillium lecanii & $\begin{array}{c}8.46 \\
\mathbf{( 2 . 9 0 )}\end{array}$ & $\begin{array}{c}4.66 \\
\mathbf{( 2 . 1 5 )}\end{array}$ & $\begin{array}{c}4.33 \\
\mathbf{( 2 . 0 8 )}\end{array}$ & $\begin{array}{c}3.13 \\
(\mathbf{1 . 7 6})\end{array}$ & $\begin{array}{c}4.04 \\
(\mathbf{2 . 0 1 )}\end{array}$ \\
\hline $\mathbf{T}_{3}$ & Metarhizium anisopliae & $\begin{array}{c}8.26 \\
(\mathbf{2 . 8 7})\end{array}$ & $\begin{array}{c}6.53 \\
(\mathbf{2 . 5 5})\end{array}$ & $\begin{array}{c}5.80 \\
(\mathbf{2 . 4 0})\end{array}$ & $\begin{array}{c}5.33 \\
(\mathbf{2 . 3 0})\end{array}$ & $\begin{array}{c}5.88 \\
(2.42)\end{array}$ \\
\hline $\mathbf{T}_{4}$ & Beauveria bassiana & $\begin{array}{c}8.26 \\
\mathbf{( 2 . 8 6 )}\end{array}$ & $\begin{array}{c}5.86 \\
\mathbf{( 2 . 4 2 )}\end{array}$ & $\begin{array}{c}5.40 \\
\mathbf{( 2 . 3 2 )}\end{array}$ & $\begin{array}{c}4.73 \\
(\mathbf{2 . 1 7})\end{array}$ & $\begin{array}{c}5.33 \\
(\mathbf{2 . 3 0})\end{array}$ \\
\hline $\mathbf{T}_{5}$ & Acetamiprid $20 \%$ SP & $\begin{array}{c}5.20 \\
(\mathbf{2 . 2 8})\end{array}$ & $\begin{array}{c}1.33 \\
(\mathbf{1 . 1 5})\end{array}$ & $\begin{array}{c}1.16 \\
\mathbf{( 1 . 0 7 )}\end{array}$ & $\begin{array}{c}0.85 \\
(\mathbf{0 . 9 2})\end{array}$ & $\begin{array}{c}1.11 \\
(\mathbf{1 . 0 5})\end{array}$ \\
\hline $\mathbf{T}_{6}$ & Imidacloprid $17.8 \% \mathrm{SL}$ & $\begin{array}{c}5.13 \\
(\mathbf{2 . 2 6})\end{array}$ & $\begin{array}{c}1.06 \\
\mathbf{( 1 . 0 3 )}\end{array}$ & $\begin{array}{c}0.93 \\
(\mathbf{0 . 9 6})\end{array}$ & $\begin{array}{c}0.73 \\
(\mathbf{0 . 8 5})\end{array}$ & $\begin{array}{c}0.91 \\
(\mathbf{0 . 9 5})\end{array}$ \\
\hline $\mathbf{T}_{7}$ & Trizophos $40 \%$ EC & $\begin{array}{c}6.00 \\
(\mathbf{2 . 4 4 )}\end{array}$ & $\begin{array}{c}2.20 \\
(\mathbf{1 . 4 8})\end{array}$ & $\begin{array}{c}1.80 \\
\mathbf{( 1 . 3 4 )}\end{array}$ & $\begin{array}{c}1.46 \\
(\mathbf{1 . 2 1})\end{array}$ & $\begin{array}{c}1.82 \\
(\mathbf{1 . 3 5})\end{array}$ \\
\hline & F- test & NS & S & S & S & S \\
\hline & S. Ed. $( \pm)$ & 0.290 & 0.049 & 0.088 & 0.056 & 0.023 \\
\hline & C. D. $(P=0.05)$ & 0.870 & 0.103 & 0.139 & 0.111 & 0.071 \\
\hline
\end{tabular}

*Figures in parenthesis are square root transformed values. DBS-Days before spray, DAS-Days after spray. 
Table.3 Effect of some bio-rational and chemical insecticides against whitefly, Bemisia tabaci (Gennadius) on okra Abelmoschus esculentus (L.) Moench (First and second spray pooled mean)

\begin{tabular}{|c|c|c|c|c|}
\hline \multirow[t]{2}{*}{ Tr. No. } & \multirow[t]{2}{*}{ Treatments } & \multicolumn{3}{|c|}{ Number of Whiteflies / 3leaves } \\
\hline & & I-Spray & II-Spray & Overall Mean \\
\hline $\mathrm{T}_{0}$ & Untreated (Control) & $\begin{array}{c}9.62 \\
(\mathbf{3 . 1 0})\end{array}$ & $\begin{array}{l}11.51 \\
(\mathbf{3 . 3 9})\end{array}$ & $\begin{array}{l}10.56 \\
(\mathbf{3 . 2 5})\end{array}$ \\
\hline $\mathrm{T}_{1}$ & Neem oil $(0.03 \%)$ & $\begin{array}{c}4.73 \\
(\mathbf{2 . 1 7})\end{array}$ & $\begin{array}{c}3.17 \\
(\mathbf{1 . 7 8})\end{array}$ & $\begin{array}{c}3.95 \\
(\mathbf{1 . 9 8})\end{array}$ \\
\hline $\mathrm{T}_{2}$ & Verticillium lecanii & $\begin{array}{c}5.40 \\
(\mathbf{2 . 3 2})\end{array}$ & $\begin{array}{c}4.04 \\
(\mathbf{2 . 0 1})\end{array}$ & $\begin{array}{c}4.72 \\
\text { (2.17) }\end{array}$ \\
\hline $\mathrm{T}_{3}$ & Metarhizium anisopliae & $\begin{array}{c}6.20 \\
(\mathbf{2 . 4 9 )}\end{array}$ & $\begin{array}{c}5.88 \\
(2.42)\end{array}$ & $\begin{array}{c}6.04 \\
(\mathbf{2 . 4 5})\end{array}$ \\
\hline $\mathrm{T}_{4}$ & Beauveria bassiana & $\begin{array}{c}5.95 \\
(\mathbf{2 . 4 4})\end{array}$ & $\begin{array}{c}5.33 \\
(\mathbf{2 . 3 0})\end{array}$ & $\begin{array}{c}5.64 \\
(2.37)\end{array}$ \\
\hline $\mathrm{T}_{5}$ & Acetamiprid $20 \%$ SP & $\begin{array}{c}2.17 \\
(\mathbf{1 . 4 7})\end{array}$ & $\begin{array}{c}1.11 \\
(\mathbf{1 . 0 5})\end{array}$ & $\begin{array}{c}1.64 \\
(\mathbf{1 . 2 8})\end{array}$ \\
\hline $\mathrm{T}_{6}$ & Imidacloprid $17.8 \% \mathrm{SL}$ & $\begin{array}{c}1.60 \\
(\mathbf{1 . 2 6})\end{array}$ & $\begin{array}{c}0.91 \\
(\mathbf{0 . 9 5})\end{array}$ & $\begin{array}{c}1.25 \\
(\mathbf{1 . 1 2})\end{array}$ \\
\hline $\mathrm{T}_{7}$ & Trizophos $40 \%$ EC & $\begin{array}{c}2.80 \\
(\mathbf{1 . 6 7})\end{array}$ & $\begin{array}{c}1.82 \\
(\mathbf{1 . 3 5})\end{array}$ & $\begin{array}{c}2.31 \\
(\mathbf{1 . 5 2})\end{array}$ \\
\hline F- test & & $\mathrm{S}$ & $\mathrm{S}$ & $\mathrm{S}$ \\
\hline S. Ed. $( \pm$ & & 0.017 & 0.023 & 0.012 \\
\hline C. D. $(\mathbf{P}$ & $0.05)$ & 0.060 & 0.071 & 0.050 \\
\hline
\end{tabular}

*Figures in parenthesis are square root transformed values. 
Table.4 Economics of cultivation

\begin{tabular}{|c|c|c|c|c|c|c|c|c|}
\hline 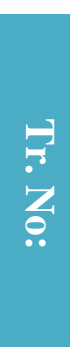 & Treatment & 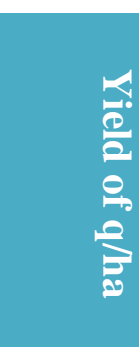 & 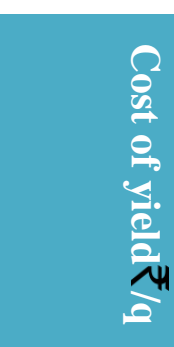 & 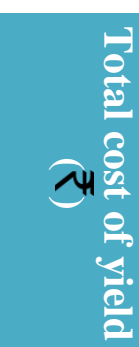 & 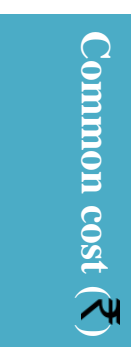 & 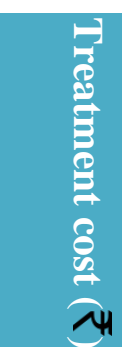 & $\frac{\overrightarrow{0}}{3}$ & $\begin{array}{l}E \\
\ddot{\theta} \\
\ddot{\theta} \\
\ddot{\theta}\end{array}$ \\
\hline$T_{0}$ & Control & 62.56 & 2000 ₹/q & 125120 & 25728 & - & 25728 & $1: 4.86$ \\
\hline$T_{1}$ & $\begin{array}{l}\text { Neem oil } \\
(0.03 \%)\end{array}$ & 98.46 & $2000 ₹ / q$ & 196920 & 25728 & 1650 & 27378 & $1: 7.19$ \\
\hline $\mathbf{T}_{2}$ & $\begin{array}{c}\text { Verticillium } \\
\text { lecanii }\end{array}$ & 81.43 & 2000₹/q & 162860 & 25728 & 9650 & 35378 & $1: 4.60$ \\
\hline $\mathbf{T}_{3}$ & $\begin{array}{c}\text { Metarhizium } \\
\text { anisopliae }\end{array}$ & 77.82 & 2000₹/q & 155640 & 25728 & 2025 & 27753 & $1: 5.60$ \\
\hline$T_{4}$ & $\begin{array}{c}\text { Beauveria } \\
\text { bassiana }\end{array}$ & 78.62 & $2000 ₹ / q$ & 157240 & 25728 & 1170 & 26898 & $1: 5.84$ \\
\hline $\mathbf{T}_{5}$ & $\begin{array}{c}\text { Acetamiprid } \\
20 \% \text { SP }\end{array}$ & 121.40 & $2000 ₹ / q$ & 242800 & 25728 & 1248 & 26976 & 1:9.00 \\
\hline$T_{6}$ & $\begin{array}{c}\text { Imidacloprid } \\
17.8 \% \text { SL }\end{array}$ & 133.80 & 2000₹/q & 267600 & 25728 & 2940 & 28668 & $1: 9.33$ \\
\hline $\mathbf{T}_{7}$ & $\begin{array}{c}\text { Trizophos } \\
\text { 40\% EC }\end{array}$ & 116.12 & $2000 ₹ / q$ & 232240 & 25728 & 1650 & 27378 & $1: 8.48$ \\
\hline
\end{tabular}

The yields among the treatment were significant. The highest yield was recorded (Table 4) in $\mathrm{T}_{6^{-}}$Imidacloprid $17.8 \% \mathrm{SL}$ (133.8q/ha), followed by $\mathrm{T}_{5}-$ Acetamiprid 20\% SP (121.4 q/ha), $\mathrm{T}_{7}$ - Triazophos $40 \%$ EC (116.12 q/ha), $\mathrm{T}_{1^{-}}$Neem oil (98.46 q/ha), $\mathrm{T}_{2^{-}}$ Verticillum lecanii $(81.43 \mathrm{q} / \mathrm{ha}), \mathrm{T}_{4}-$ Beauveria bassiana (78.62 q/ha), $\mathrm{T}_{3^{-}}$ Metarhizium anisopliae $(77.82 \mathrm{q} / \mathrm{ha})$, as compared to control $\mathrm{T}_{0^{-}}(62.56 \mathrm{q} / \mathrm{h})$. When benefit cost ratio was worked out, interesting result was achieved. Among the treatment studied, the best and most economical treatment was $\mathrm{T}_{6^{-}}$Imidacloprid $17.8 \% \mathrm{SL}$ (1:9.33), followed by $\mathrm{T}_{5}-$ Acetamiprid $20 \%$ SP (1:9.00), $\mathrm{T}_{7}$ - Triazophos 40\% EC (1:8.48), $\mathrm{T}_{1}$ - Neem oil (1:7.19), $\mathrm{T}_{2}$ - Verticillum lecanii (1:4.60), $\mathrm{T}_{4}$ - Beauveria bassiana (1:5.84), $\mathrm{T}_{3}-$ Metarhizium anisopliae (1:5.60), as compared to control $\mathrm{T}_{0^{-}}(1: 4.86)$.
Simlar readings were found with, Naik and Shekarappa (2009) who reported that, the treatments Metarhizium anisopliae (38.80 $\mathrm{q} / \mathrm{ha}$ ) and Verticillium lecanii (38.50 q/ha) recorded with returns of 1:18.40 and 1:16.68 respectively. These findings can be supported with the study of Parmar et al., (2013) who reported that from the experiment, the treatment Imidacloprid produced highest $(105.22 \mathrm{q} / \mathrm{ha})$ yield with (1:22.08) Benefit Cost Ratio.

From the critical analysis of the present findings it can be concluded that, Insecticides like Imidacloprid 17.8\% SL and Acetamiprid $20 \%$ SP, Triazophos $40 \%$ EC can be suitably incorporated in pest management schedule against Bemisia tabaci (Gennadius) as an effective tool under chemical control, and treatments like Neem oil, Verticillum lecanii, 
Beauveria bassiana and Metarhizium anisopliae are also to be incorporated in pest management in order to avoid indiscriminate use of pesticides causing pollution in the environment and not much harmful to beneficial insects and in increasing cost effectiveness.

\section{References}

Aykroud, W. R. (1963). I.C.M.R. Special report series. 42.

Begum, K. R., Patil, S. and Mohite, P. (2016). Evaluation of Newer Molecules of Insecticides against Sucking Pests complex Infesting Okra. Ind. J. Appl. Sci., 6(2): 30-34.

Chaudhary, H. R. and Dadeech (1989). Incidence of insects attacking okra and the available losses caused by them. Ann. Arid Zone, 28(3): 305-307.

Chauhan, D. V. S. (1972). Vegetable production in India. 3rd Ed., Pub. Ram Prasad and Sons, Agra.

Ghosh, S. K., Mandal, T. and Chakraborty, K. (2013). Efficacy of chemical insecticides and neem oil against whitefly (Bemisia tabaci Genn.) infesting ladysfinger (Abelmoschus esculentus L.). Int. J. of Bio-resou and Stres. Manag., 4(2): 348-351.

Naik, P. R. H. and Shekharappa (2009).Field evaluation of different entomopathogenic fungal formulations against sucking pests of okra. Karnataka J. Agric. Sci., 22(3):575-578.

Parmar, K. D., Korat, D. M., Joshi, M. N., Patel, A. R. and Shah, P. G. (2013).Relative bio-efficacy of insecticides/miticides against pest complex of okra. Karnataka J. Agric. Sci., 26(3): 375-378.

Preetha, G., Manoharan, T., Stanley, J. and Kuttalam, S. (2009). Evaluation of imidacloprid against okra jassid, Amrasca biguttula biguttula (Ishida) and whitefly, Bemisia tabaci (Gennadius). Ind. J. Ento., 71(3): 209214.

Sarkar, S., Patra, S. and Samanta, A. (2016). Efficacy of different bio-pesticides against sucking pests of okra (Abelmoschus esculentus L. Moench). J. of App. and Nat. Sci., 8(1): 333-339.

\section{How to cite this article:}

Sumit Kumar Singh and Sasya Thakur. 2018. Comparative Efficacy of Some Bio-Rational and Chemical Insecticides against Whitefly Bemisia tabaci (Gennadius) on Okra Abelmoschus esculentus (L.) Moench. Int.J.Curr.Microbiol.App.Sci. 7(08): 4405-4411. doi: https://doi.org/10.20546/ijcmas.2018.708.463 